\title{
Effectiveness and tolerability of second-line treatment with vildagliptin versus other oral drugs for type 2 diabetes in a real-world setting in the Middle East: results from the EDGE study
}

\author{
This article was published in the following Dove Press journal: \\ Vascular Health and Risk Management \\ 24 February 2015 \\ Number of times this article has been viewed
}

\author{
Charles Saab' \\ Feryal A Al-Saber ${ }^{2}$ \\ Jihad Haddad ${ }^{3}$ \\ Mahir Khalil Jallo ${ }^{4}$ \\ Habib Steitieh ${ }^{5}$ \\ Giovanni Bader ${ }^{6}$ \\ Mohamed Ibrahim ${ }^{7}$ \\ 'Department of Endocrinology and \\ Metabolism, Sacre Coeur University \\ Hospital, Baabda, Lebanon; ${ }^{2}$ Endocrine \\ Department, Bahrain Defence Force \\ Hospital, Rifaa, Bahrain; ${ }^{3}$ Division \\ of Endocrinology Department of \\ Internal Medicine, Prince Hamaza \\ Hospital, Amman, Jordan; ${ }^{4}$ Department \\ of Internal Medicine, Gulf Medical \\ University, Ajman, United Arab \\ Emirates; ${ }^{5}$ New Mowasat Hospital, \\ Safat, Kuwait; ${ }^{6}$ Novartis Pharma AG, \\ Basel, Switzerland; ${ }^{7}$ Novartis Pharma \\ Services AG, Dubai, United Arab \\ Emirates
}

Background: Type 2 diabetes mellitus (T2DM) is a chronic progressive disease that requires treatment intensification with antihyperglycemic agents due to progressive deterioration of $\beta$-cell function. A large observational study of 45,868 patients with T2DM across 27 countries (EDGE) assessed the effectiveness and safety of vildagliptin as add-on to other oral antidiabetic drugs (OADs) versus other comparator OAD combinations. Here, we present results from the Middle East countries (Bahrain, Jordan, Kuwait, Lebanon, Oman, Palestine, and the United Arab Emirates).

Methods: Patients inadequately controlled with OAD monotherapy were eligible after the add-on treatment was chosen by the physician based on clinical judgment and patient need. Patients were assigned to either vildagliptin or comparator OADs (sulfonylureas, thiazolidinediones, glinides, $\alpha$-glucosidase inhibitors, or metformin, except incretin-based therapies) based on the add-on therapy. The primary endpoint was the proportion of patients achieving a glycated hemoglobin $\left(\mathrm{HbA}_{1 \mathrm{c}}\right)$ reduction of $>0.3 \%$ without peripheral edema, hypoglycemia, discontinuation due to a gastrointestinal event, or weight gain $\geq 5 \%$. One of the secondary endpoints was the proportion of patients achieving $\mathrm{HbA}_{1 \mathrm{c}}<7 \%$ without hypoglycemia or weight gain. Change in $\mathrm{HbA}_{1 \mathrm{c}}$ from baseline to study endpoint and safety were also assessed.

Results: Of the 4,780 patients enrolled in the Middle East, 2,513 received vildagliptin and 2,267 received other OADs. Overall, the mean ( \pm standard deviation) age at baseline was $52.1 \pm 10.2$ years, mean $\mathrm{HbA}_{1 \mathrm{c}}$ was $8.5 \% \pm 1.3 \%$, and mean $\mathrm{T} 2 \mathrm{DM}$ duration was $4.2 \pm 4.0$ years. The proportion of patients achieving the primary $(76.1 \%$ versus $61.6 \%, P<0.0001)$ and secondary (54.8\% versus $29.9 \%, P<0.0001)$ endpoints was higher with vildagliptin than with the comparator OADs. The unadjusted odds ratios for the primary and secondary endpoints were 1.98 (95\% confidence interval 1.75-2.25) and 2.8 (95\% confidence interval 2.5-3.2), respectively, in favor of vildagliptin. Vildagliptin achieved a numerically greater reduction in $\mathrm{HbA}_{1 \mathrm{c}}(1.7 \%)$ from baseline versus comparator OADs (1.4\%). The overall incidence of adverse events was comparable between studied cohorts.

Conclusion: In real life, treatment with vildagliptin was associated with a higher proportion of patients with T2DM achieving better glycemic control without tolerability issues in the Middle East.

Keywords: dipeptidyl peptidase-4, Middle East, oral antidiabetic drugs, real world, type 2 diabetes mellitus, vildagliptin

\section{Introduction}

In recent years, type 2 diabetes mellitus (T2DM) has emerged as a global epidemic, which is predicted to worsen in the coming decades, particularly in developing countries.
Correspondence: Mohamed Ibrahim Novartis Pharma Services AG, MENA Cluster, PO Box 23510 Dubai, United Arab Emirates

Tel +97I 44357038

Fax +97| 4435 770 I

Email mohamed-I.ibrahim@novartis.com 
Rapid urbanization, changes in nutrition, dietary patterns, sedentary habits, and obesity have all contributed to the progression of the epidemic in the Middle East. ${ }^{1}$ The current prevalence of diabetes in the Middle East and North Africa (MENA) region is estimated to be $9.2 \%$, which is equivalent to 34.6 million people with diabetes. This figure is expected to double by 2035 to 67.9 million. ${ }^{2}$ Nearly half of the people with T2DM remain undiagnosed, and in those who are diagnosed, glycemic control is suboptimal, which potentially increases the risk of diabetes complications and results in poor health outcomes. ${ }^{2}$ A systematic review of a subset of countries in the Gulf region, which summarized the findings of 27 studies, reported that $<50 \%$ of people with diabetes reached their targets for the management of risk factors such as glycemic control, hypertension, and dyslipidemia, ${ }^{3}$ suggesting the need for implementation of strategies for prevention, early detection, and consistent management of T2DM. This further underscores the need for developing countries to review, update, and implement new national diabetes programs that will help access the effectiveness of newer therapies and their cost-effectiveness.

Current treatment guidelines for management of T2DM recommend a patient-centered approach taking into account factors such as effectiveness, tolerability, long-term safety, cost, and patient preferences when choosing antihyperglycemic agents. ${ }^{4}$ T2DM is a chronic progressive disease, so often requires treatment intensification with multiple oral antidiabetic drugs (OADs) that have complementary mechanisms of action to maintain glycemic control and prevent longterm complications. ${ }^{4}$ However, there is limited information available on the effectiveness of newer therapeutic alternatives for diabetes, such as dipeptidyl peptidase-4 (DPP-4) inhibitors, in the Middle Eastern population, possibly due to under-representation of patients from this region in large randomized global studies. Thus, real-life data are needed to understand the impact of differences in environmental, genetic, and physical factors and clinical practice in the overall response to newer therapies and further management of T2DM in the Middle Eastern population. Vildagliptin is a potent and selective DPP-4 inhibitor that improves glycemic control by increasing $\alpha$-cell and $\beta$-cell responsiveness to glucose and has been shown to be weight neutral with no additional risk for hypoglycemia. ${ }^{5-7}$ The efficacy and safety of vildagliptin both as monotherapy and in combination with other OADs has been demonstrated in randomized clinical trials. ${ }^{7-10}$

We performed a post hoc analysis of the EDGE (Effectiveness of Diabetes control with vildaGliptin and vildagliptin/mEtformin) study to evaluate the effectiveness and tolerability of add-on vildagliptin compared with any other OADs added to monotherapy in real-life clinical practice, which provided us with an opportunity to look into the regional perspectives of real-life management of T2DM. ${ }^{11}$ Here we present the results from the EDGE study for participants from seven countries in the Middle East (Jordan, Palestine, Oman, Bahrain, Kuwait, Lebanon, and the United Arab Emirates). Three of the seven countries included in the present analysis (Bahrain, Kuwait, and the United Arab Emirates) are among the top 15 countries in the world with respect to national prevalence of diabetes. ${ }^{12}$

\section{Materials and methods Study design and patient population}

The EDGE study enrolled 45,868 people with T2DM across 27 countries from Europe, East Asia, India, Latin America, and the Middle East. Details of the study population and design are reported elsewhere. ${ }^{11}$ In brief, people with T2DM aged $\geq 18$ years, taking OAD monotherapy, and prescribed a new add-on OAD by the physician at their discretion based on patient need were enrolled in the study. Participants were assigned to one of two cohorts (Figure 1) to receive vildagliptin or comparator OADs (metformin, sulfonylureas, thiazolidinediones, glinides, $\alpha$-glucosidase inhibitors) as add-on to monotherapy. People who were prescribed DPP-4 inhibitors other than vildagliptin or glucagon-like peptide-1 mimetics/ analogs were excluded. All participants provided informed consent before enrolling in the study. To avoid physician bias for a particular choice of antihyperglycemic agent, patients were enrolled in the study only after they had received the prescription for an OAD. The term "index therapy" is used to represent the combination treatment initiated at enrollment. For any index therapy, a fixed-dose combination was allowed, if available.

\section{Study assessments}

The primary endpoint was the proportion of participants responding to treatment with an $\mathrm{HbA}_{1 \mathrm{c}}$ reduction $>0.3 \%$ from baseline to study endpoint with no tolerability findings such as peripheral edema, hypoglycemia, discontinuation due to a gastrointestinal event, or weight gain $\geq 5 \%$. One of the secondary endpoints was the responder rate, measured as the proportion of participants achieving target $\mathrm{HbA}_{1 \mathrm{c}}<7.0 \%$ at the end of study without hypoglycemia or weight gain $\geq 3 \%$ in participants with baseline $\mathrm{HbA}_{1 \mathrm{c}} \geq 7 \%$. The change in $\mathrm{HbA}_{1 \mathrm{c}}$ from baseline to study endpoint (analysis not prespecified in the protocol) and the number of hypoglycemic events were also evaluated in this post hoc analysis. Hypoglycemia was 


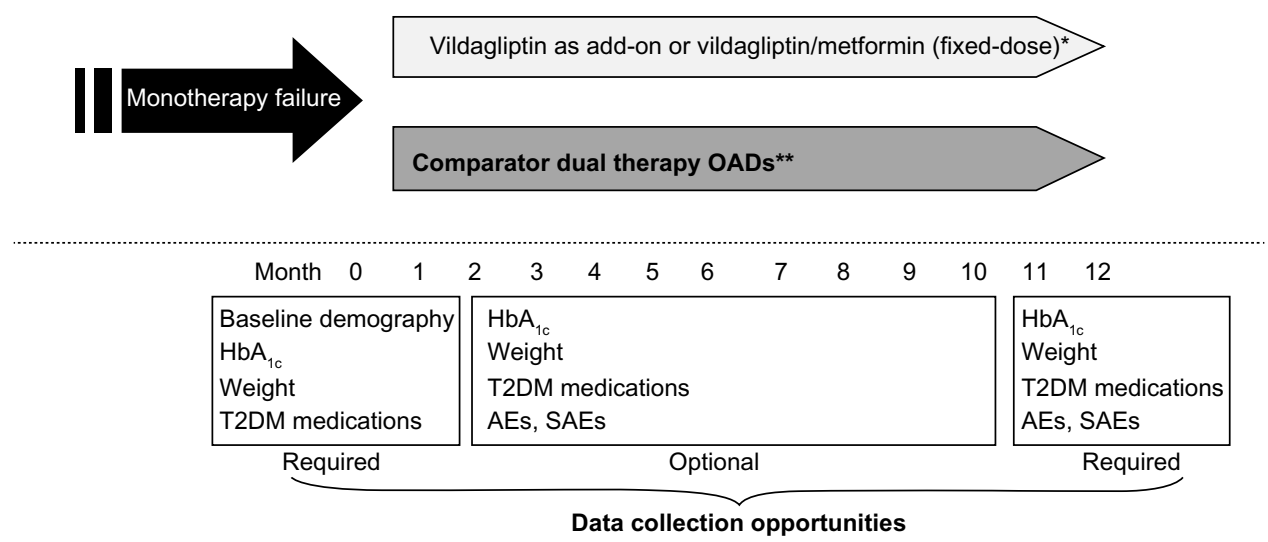

Figure I Study design.

Notes: *Vildagliptin cohort includes people with T2DM newly initiating vildagliptin as add-on dual OAD therapy, or newly initiating vildagliptin-metformin (fixed dose) from non-vildagliptin monotherapy; **comparator OAD cohort includes people with T2DM newly initiating dual OAD therapy with antidiabetic therapies other than vildagliptin (defined as sulfonylureas, metformin, thiazolidinediones, metiglinides, or $\alpha$-glucosidase inhibitors as add-on dual therapy) except as add-on to vildagliptin, other dipeptidyl peptidase-4 inhibitors, or glucagon-like peptide-I mimetics/analogs.

Abbreviations: AEs, adverse events; OADs, oral anti-diabetic drugs; SAEs, serious adverse events; T2DM, type 2 diabetes mellitus.

defined as symptoms suggestive of hypoglycemia including mild and severe events that resolved promptly after administration of an oral carbohydrate.

\section{Statistical analysis}

Descriptive statistics were used for these regional post hoc analyses. Baseline demographics and safety data are reported for the intent-to-treat population that comprised participants newly receiving dual therapy including vildagliptin or other OADs, except incretin-based therapies, added to prior monotherapy. The analyses for the primary and secondary composite endpoints utilized the per protocol population, which was a subset of the intent-to-treat population without protocol deviations. Data were censored if participants changed their index therapy. A binary logistic regression model was used to calculate the odds ratios (ORs) with $95 \%$ confidence intervals (CIs) for the probability of success in achieving the primary and secondary endpoints. The OR expresses odds in favor of success with vildagliptin combinations relative to odds in favor of success with comparator OADs. Participants whose outcomes could not be categorized as a success or failure (eg, due to missing $\mathrm{HbA}_{1 \mathrm{c}}$ or body weight data at the 12-month endpoint) were considered nonevaluable and not included in the calculation of the OR. Only unadjusted ORs are reported for the primary and secondary endpoints. Change in $\mathrm{HbA}_{1 \mathrm{c}}$ was adjusted with respect to baseline value by using the analysis of covariance model. The change in $\mathrm{HbA}_{1 \mathrm{c}}$ from baseline to study endpoint was calculated for the intent-to-treat population; however, the statistical significance between cohorts was not assessed because the analysis was not prespecified in the protocol.
The protocol for the EDGE study was approved by all local independent review boards or ethics committees.

\section{Results}

Of the 4,780 participants enrolled in the Middle East, 2,513 participants were assigned to the vildagliptin cohort and 2,267 participants were assigned to the comparator cohort (Table 1). Despite the non-randomized nature of the study, the cohorts were well balanced for demographic and baseline characteristics. Overall, on average, the participants were 52 years of age, with a body mass index of $29.4 \mathrm{~kg} / \mathrm{m}^{2}, \mathrm{HbA}_{1 \mathrm{c}}$ $8.5 \%$, and a T2DM duration of 4.2 years at monotherapy failure (Table 2). Although the baseline demographics were well balanced between the two cohorts, there were small but statistically significant differences observed between age, body mass index, and duration of diabetes. Participants in the

Table I Patient population and flow

\begin{tabular}{lll}
\hline Enrolled* $(\mathbf{n = 4 , 7 8 0})$ & $\begin{array}{l}\text { Vildagliptin } \\
\text { cohort }\end{array}$ & $\begin{array}{l}\text { Comparator } \\
\text { cohort }\end{array}$ \\
\hline Assigned & 2,513 & 2,267 \\
No adequate source & 0 & $\mathrm{I}$ \\
$\begin{array}{l}\text { documentation at site; lack of } \\
\text { quality and accuracy of data entry }\end{array}$ & & \\
$\begin{array}{l}\text { Intent to treat** } \\
\text { Completed }\end{array}$ & 2,513 & 2,266 \\
Discontinued & 2,390 & 2,137 \\
Per protocol*** & 123 & 129 \\
\hline
\end{tabular}

Notes: *Enrolled population includes all patients who gave documented informed consent; **intent-to-treat population is a subset of the enrolled population and includes all patients who were assigned to new treatment at study start; *** per protocol population is a subset of the intent-to-treat population which completed the study without any major protocol deviation. It was used for analysis of effectiveness endpoints. 
Table 2 Demographic and patient baseline characteristics (intent-to-treat population)

\begin{tabular}{|c|c|c|c|c|}
\hline Characteristic & Vildagliptin & Comparator & Total & $P$-value \\
\hline Mean \pm SD or $n(\%)$ & $n=2,513$ & $n=2,266$ & $n=4,779$ & \\
\hline Age (years) & $51.4 \pm 10.10$ & $52.8 \pm 10.33$ & $52.1 \pm 10.23$ & 0.000 \\
\hline Sex, male & $1,599(63.6)$ & I,343 (59.3) & $2,942(61.6)$ & 0.002 \\
\hline BMI $\left(\mathrm{kg} / \mathrm{m}^{2}\right)$ & $29.7 \pm 4.8$ & $29.1 \pm 4.6$ & $29.4 \pm 4.7$ & 0.000 \\
\hline $\mathrm{HbA}_{\mathrm{lc}}(\%)$ & $8.5 \pm 1.2$ & $8.5 \pm 1.3$ & $8.5 \pm 1.3$ & 0.045 \\
\hline Duration of T2DM (years) & $4.0 \pm 4.0$ & $4.4 \pm 4.0$ & $4.2 \pm 4.0$ & 0.000 \\
\hline
\end{tabular}

Abbreviations: $\mathrm{BMI}$, body mass index; $\mathrm{HbA}_{\mathrm{lc}}$, glycated hemoglobin; SD, standard deviation; T2DM, type 2 diabetes mellitus.

comparator cohort were on average 1.4 years older $(P=0.000)$ and had a longer duration of T2DM by 0.4 years $(P=0.000)$ when compared with the vildagliptin cohort. Table 3 reports index therapies in the intent-to-treat population for both cohorts. At study entry, most participants $(\sim 86 \%)$ were receiving metformin monotherapy, followed by sulfonylureas $(\sim 11 \%)$, thiazolidinediones $(\sim 2.0 \%)$, glinides and alphaglucosidase inhibitors (1.0\% each).

\section{Efficacy}

The primary endpoint was reached in $76.1 \%$ of participants in the vildagliptin cohort versus $61.6 \%$ of participants in the comparator OAD cohort, with an unadjusted OR of 1.98 (95\% CI 1.75-2.25; $P<0.0001)$ in favor of vildagliptin. Likewise, the responder rate for the secondary endpoint (achieving $\mathrm{HbA}_{1 \mathrm{c}}<7.0 \%$ without hypoglycemia or weight gain $\geq 3 \%$ in those patients with baseline $\mathrm{HbA}_{1 \mathrm{c}} \geq 7 \%$ ) was higher in the vildagliptin cohort compared with the comparator OAD cohort ( $54.8 \%$ versus $29.9 \%$, respectively), resulting in an unadjusted OR of $2.8(95 \%$ CI $2.5-3.2 ; P<0.0001)$ in

Table 3 Index medication (intent-to-treat population)

\begin{tabular}{|c|c|c|c|}
\hline \multicolumn{2}{|c|}{ Vildagliptin cohort $(n=2,5 \mid 3)^{*}$} & \multicolumn{2}{|c|}{ Comparator cohort $(n=2,266) *$} \\
\hline Treatments & $\begin{array}{l}\text { Participants, } \\
\text { n (\%) }\end{array}$ & Treatments & $\begin{array}{l}\text { Participants, } \\
\text { n (\%) }\end{array}$ \\
\hline $\begin{array}{l}\text { Metformin- } \\
\text { vildagliptin }\end{array}$ & $2,028(80.8)$ & Metformin-SU & I,686 (74.5) \\
\hline SU-vildagliptin & $399(15.9)$ & Metformin-TZD & $328(14.5)$ \\
\hline TZD-vildagliptin & $50(2.0)$ & SU-TZD & $128(5.7)$ \\
\hline $\begin{array}{l}\text { Glinide- } \\
\text { vildagliptin }\end{array}$ & $26(1.0)$ & $\begin{array}{l}\text { Glinide- } \\
\text { metformin }\end{array}$ & $68(3.0)$ \\
\hline \multirow[t]{6}{*}{ AGI-vildagliptin } & $7(0.3)$ & AGI-metformin & $27(1.2)$ \\
\hline & & AGI-SU & $12(0.5)$ \\
\hline & & Glinide-SU & $6(0.3)$ \\
\hline & & AGI-TZD & $4(0.2)$ \\
\hline & & Glinide-TZD & $4(0.2)$ \\
\hline & & AGI-glinide & I $(0.04)$ \\
\hline
\end{tabular}

Notes: *Data for three patients in the vildagliptin cohort and two patients in the comparator cohort are missing. Percentages are calculated based on nonmissing values.

Abbreviations: AGI, alpha glucosidase inhibitor; SU, sulfonylurea; TZD, thiazolidinedione. favor of vildagliptin (Figure 2A). After 12 months of treatment, mean $\mathrm{HbA}_{1 \mathrm{c}}$ decreased from baseline in both cohorts ( $-1.7 \%$ with vildagliptin versus $-1.4 \%$ with comparators), with a between-group difference of $-0.3 \%$ (Figure $2 \mathrm{~B}$ ).

\section{Safety}

Overall, 87 patients $(3.5 \%)$ in the vildagliptin cohort and 135 patients $(6.0 \%)$ in the comparator cohort reported adverse events. The occurrence of serious adverse events was low in
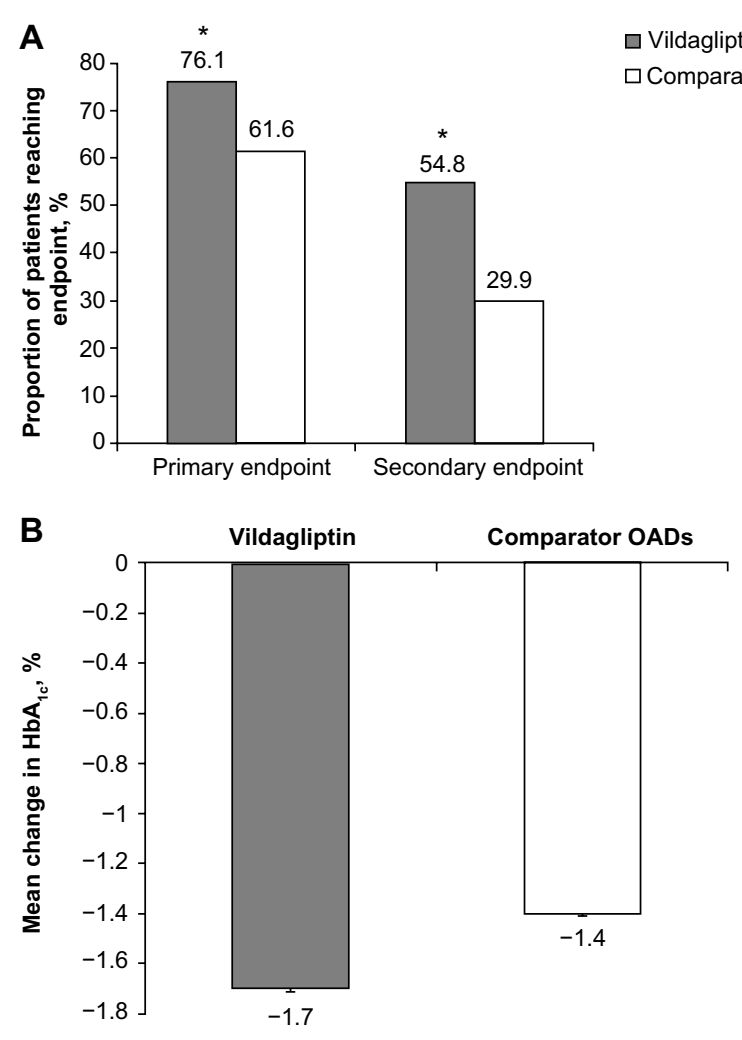

Figure 2 (A) Proportion of participants achieving study endpoints in per protocol population. (B) Mean ( \pm standard error) change in $\mathrm{HbA}_{\mathrm{lc}}$ from baseline to study endpoint in intent-to-treat population using an adjusted analysis of covariance method. The primary endpoint was the proportion of patients experiencing an $\mathrm{HbA}_{\mathrm{lc}}$ decrease of $>0.3 \%$ without hypoglycemia, weight gain, peripheral edema, or gastrointestinal side effects. $* P<0.000$ I for unadjusted odds ratio of 1.98 ( $95 \%$ confidence interval 1.75-2.25). The secondary endpoint was the proportion of patients reaching $\mathrm{HbA}_{\mathrm{lc}}<7 \%$ with no hypoglycemic events and weight gain. $* P<0.000$ I for unadjusted odds ratio of 2.8 (95\% confidence interval 2.5-3.2).

Abbreviations: $\mathrm{HbA}_{\mathrm{lc}}$, glycated hemoglobin; OADs, oral antidiabetic drugs. 
both the cohorts; two patients $(0.1 \%)$ in each cohort reported serious adverse events, and none of them were considered to be related to the study drug. No death occurred in either cohort. The incidence of hypoglycemia was low at $0.4 \%$ and $2.3 \%$ in the vildagliptin and comparator OAD cohorts, respectively.

\section{Discussion}

The increasing prevalence of T2DM in the Middle East, especially among the younger age groups, is worrying; half of all deaths from diabetes in the MENA region have been reported to occur in people aged $<60$ years. ${ }^{12}$ Further, there are limited data on glycemic control and management of T2DM for newer therapies in the region. Thus, more reallife studies that provide an evidence base for the long-term management of T2DM are warranted. The present post hoc analysis of the EDGE study in the Middle East regarding the effectiveness and tolerability of vildagliptin as add-on to another OAD compared with all other OAD combinations in people with T2DM will complement the data from randomized controlled trials. The baseline $\mathrm{HbA}_{1 \mathrm{c}}$ in the present post hoc analysis in the Middle East was high (8.5\%) confirming the expected high prevalence of inadequate glycemic control in this region, despite continuous efforts to raise awareness of adequate glycemic control in order to prevent the complications of diabetes. ${ }^{12}$ The mean baseline $\mathrm{HbA}_{1 \mathrm{c}}$ in the Middle East was higher than the global average in the EDGE study (8.2\%); however, it is similar to the mean baseline $\mathrm{HbA}_{1 \mathrm{c}}$ observed in Latin America (8.5\%) and India $(8.6 \%) .{ }^{13,14}$ Changes in dietary habits, physical inactivity, sedentary lifestyle, urbanization, smoking, educational level, and lack of awareness about the disease and its long-term complications have been reported as factors contributing to the suboptimal glycemic control in this region. ${ }^{15,16}$ After one year of treatment, more participants in the vildagliptin cohort achieved the primary endpoint of an $\mathrm{HbAlc}$ decrease $.0 .3 \%$, with no associated tolerability findings; than in the comparator OAD cohort. The vildagliptin cohort also showed a greater responder rate (proportion of participants achieving $\mathrm{HbA}_{1 \mathrm{c}}<7.0 \%$ without hypoglycemia or weight gain $\geq 3 \%$ in participants with baseline $\mathrm{HbA}_{1 \mathrm{c}} \geq 7 \%$ ) than the comparator OAD cohort. Tolerability factors such as body weight, hypoglycemia, and gastrointestinal events have driven the non-response to treatment in the comparator cohort (data not shown), which could be correlated with the maximum number of patients $(\sim 80 \%)$ taking sulfonylureas. These findings are similar to the proportion of patients achieving primary and secondary endpoints with vildagliptin in other regions/countries like Latin America, India, and the 12 participating European countries in the study (including Germany), despite the diverse representation of populations from these regions. ${ }^{13,14,17,18}$ The $\mathrm{HbA}_{1 \mathrm{c}}$ reduction in the present post hoc analysis was numerically higher in the vildagliptin cohort than in the comparator OAD cohort. The proportion of participants reaching the primary and secondary endpoints, as well as the $\mathrm{HbA}_{1 \mathrm{c}}$ reduction observed in the present study, were slightly higher than the findings from the overall EDGE study. ${ }^{11}$ Indeed, recently published data suggest that the efficacy of comparators, such as sulfonylureas, might be diminished in a real-life setting versus randomized controlled trials, indicating that the maximal doses of comparator OADs, sulfonylureas in particular, were not always utilized, due at least in part to concern about potential side effects. ${ }^{19}$

The high $\mathrm{HbA}_{1 \mathrm{c}}$ reductions seen in the present analysis might be due to the high baseline $\mathrm{HbA}_{1 \mathrm{c}}$ of participants in the Middle East region. Shorter duration of T2DM and young age of participants have been associated with better glycemic control in previous studies; ${ }^{20,21}$ this may also partly explain the higher responder rate in the present analysis.

Overall, the present safety/tolerability findings are in line with data from the clinical studies of vildagliptin showing no new safety signals, this includes no new cardiovascular, cerebrovascular, pancreatic, hepatic, immune system, or skin disorders. ${ }^{22,23}$ The incidence of hypoglycemia was low in the vildagliptin cohort versus the comparator OAD cohort, which is consistent with the results of an earlier study showing a similar reduced frequency of hypoglycemia with vildagliptin compared with sulfonylureas in a real-life setting. ${ }^{24}$ Further, these results are consistent with those of an observational study conducted in Asia and the Middle East in patients fasting during Ramadan which showed a low incidence of hypoglycemia with vildagliptin versus sulfonylureas. ${ }^{25,26}$ The low risk of hypoglycemia seen with vildagliptin is also of particular interest in the Middle East region, which has a largely Muslim population that fasts during the holy month of Ramadan. There is a high likelihood that a significant patient population from the Middle East region participating in the 12-month EDGE study might have fasted safely during Ramadan with no additional risk of hypoglycemia. The low incidence of hypoglycemia observed might be explained by vildagliptin's effect of increasing glucose-dependent insulinotropic polypeptide levels between meals and overnight, when hypoglycemia is most likely to occur, thereby increasing glucagon levels in hypoglycemia. ${ }^{10}$

Our study has certain limitations. Due to its open-label design, physicians could have selected any drug based on 
their clinical judgment, resulting in an imbalance in the treatment arms, favoring the newly introduced alternative agent, vildagliptin, in the overall EDGE study. However, in the present analysis, both cohorts seem to be balanced despite the open-label design. Further, in controlled clinical trial settings, reporting of adverse events is done in a systematic manner on dedicated forms, but in the present observational study was done based on a voluntary reporting system, which might have resulted in under-reporting of events. Despite its limitations, the EDGE study mapped real-life clinical practice in the Middle East with lifestyle interventions followed by metformin being prescribed as first-line therapy followed by treatment intensification if patients were not at goal, so the findings are reflective of real-life T2DM management.

In conclusion, the results of this post hoc analysis of real-life clinical practice in the Middle East demonstrate that, in people with T2DM in whom second-line oral glucoselowering therapy was initiated, vildagliptin resulted in lowering $\mathrm{HbA}_{1 \mathrm{c}}$ to $<7.0 \%$ without weight gain, hypoglycemia, or peripheral edema, despite the delayed intensification of treatment with second-line OAD as suggested by a high baseline $\mathrm{HbA}_{1 \mathrm{c}}$ at time of enrollment. Vildagliptin has a good safety profile and was generally well tolerated.

\section{Acknowledgments}

The authors gratefully acknowledge the Middle Eastern physicians and volunteers who participated in this study. The authors would also like to thank Päivi M Paldánius, Global Medical Affairs Diabetes (Novartis Pharma AG, Basel, Switzerland) for her valuable input and suggestions while drafting the manuscript, and G Lakshmi Deepa, Novartis Healthcare Private Limited, India, for medical writing support.

\section{Author contributions}

CS, FAAS, JH, MKJ, and HS all contributed to the conduct of the study and reviewed and approved the manuscript for submission. GB was involved in the data analysis and both MI and GB were involved in interpretation of the data, and review and approval of the manuscript for submission. In addition, all authors meet authorship requirements and agree to be accountable for all aspects of the work.

\section{Disclosure}

This study was funded by Novartis Pharma AG, Basel, Switzerland. CS has received research honoraria, participated in advisory boards, and served as a speaker for AstraZeneca/ Algorithm, Servier, Merck, Sharpe and Dohme, Novo Nordisk, Benta, Novartis, Sanofi Aventis, Roche, Genentech,
Merck-Serono, Ares-Serono, Chiesi, Pfizer, Eli Lilly, Boehringer Ingelheim, and Johnson and Johnson. FAAS has received research honoraria, participated in advisory boards, and served as a speaker for AstraZeneca, Servier, Merck, Sharpe and Dohme, Novo Nordisk, and Novartis. JH has participated in an advisory panel for Novo Nordisk and been a speaker for Merck Sharp and Dohme, Merck Serono, Novo Nordisk, Novartis, AstraZeneca, Menarini, and Boehringer Ingelheim. KJM has received research honoraria from Novartis, Sanofi, Eli Lilly, and NovoNordisk, and served on consultancy and advisory boards for AstraZeneca and Novo Nordisk. He has been speaker for Novo Nordisk, AstraZeneca, Bristol-Myers Squibb, Eli Lilly, Merck Sharp and Dohme, and Novartis. HS has received research honoraria from Novartis, Servier and Merck Sharp and Dohme. MI and GB are employees of Novartis.

\section{References}

1. Whiting DR, Guariguata L, Weil C, Shaw J. IDF Diabetes Atlas: global estimates of the prevalence of diabetes for 2011 and 2030. Diabetes Res Clin Pract. 2011;94:311-321.

2. Majeed A, El-Sayed AA, Khoja T, Alshamsan R, Millett C, Rawaf S. Diabetes in the Middle-East and North Africa: an update for 2013 for the IDF Diabetes Atlas. Diabetes Res Clin Pract. 2014;103:218-222.

3. Alhyas L, McKay A, Balasanthiran A, Majeed A. Quality of type 2 diabetes management in the states of the Cooperation Council for the Arab States of the Gulf: a systematic review. PLoS One. 2011;6:e22186.

4. Inzucchi SE, Bergenstal RM, Buse JB, et al. Management of hyperglycemia in type 2 diabetes: a patient-centered approach. Diabetes Care. 2012;35:1364-1379.

5. Balas B, Baig MR, Watson C, et al. The dipeptidyl peptidase IV inhibitor vildagliptin suppresses endogenous glucose production and enhances islet function after single dose administration in type 2 diabetic patients. J Clin Endocrinol Metab. 2007;92:1249-1255.

6. Foley JE, Jordan J. Weight neutrality with the DPP-4 inhibitor, vildagliptin: mechanistic basis and clinical experience. Vasc Health Risk Manag. 2010;6:541-548.

7. Ahren B, Foley JE, Bosi E. Clinical evidence and mechanistic basis for vildagliptin's action when added to metformin. Diabetes Obes Metab. 2011;13:193-203.

8. Matthews DR, Dejager S, Ahren B, et al. Vildagliptin add-on to metformin produces similar efficacy and reduced hypoglycaemic risk compared with glimepiride, with no weight gain: results from a 2-year study. Diabetes Obes Metab. 2010;12:780-789.

9. Ferrannini E, Fonseca V, Zinman B, et al. Fifty-two-week efficacy and safety of vildagliptin vs glimepiride in patients with type 2 diabetes mellitus inadequately controlled on metformin monotherapy. Diabetes Obes Metab. 2009;11:157-166.

10. Ahren B, Schweizer A, Dejager S, Villhauer EB, Dunning BE, Foley JE. Mechanisms of action of the dipeptidyl peptidase-4 inhibitor vildagliptin in humans. Diabetes Obes Metab. 2011;13:775-783.

11. Mathieu C, Barnett AH, Brath H, et al. Effectiveness and tolerability of oral glucose-lowering therapies in type 2 diabetes: a real-life worldwide observational study (EDGE). Int J Clin Pract. 2013;67:947-956.

12. International Diabetes Federation 2013. IDF Diabetes Atlas. 6th ed. Available from: http://www.idf.org/diabetesatlas/data-visualisations. Accessed March 4, 2014.

13. Mendivil CO, Márquez-Rodríguez E, Angel ID, et al. Comparative effectiveness of vildagliptin in combination with other oral anti-diabetes agents in usual-care conditions: the EDGE-Latin America study. Curr Med Res Opin. 2014;30:1769-1776. 
14. Wangnoo SK, Bader G, Gawai A, Singh S. Effectiveness and tolerability of vildagliptin in Indian patients with type 2 diabetes mellitus: results from EDGE - a real-world observational study. Indian J Clin Pract. 2013;24:537-642.

15. Zabetian A, Keli HM, Echouffo-Tcheugui JB, Narayan KM, Ali MK. Diabetes in the Middle East and North Africa. Diabetes Res Clin Pract. 2013;101:106-122.

16. Al-Maskari F, El-Sadig M, Al-Kaabi JM, et al. Knowledge, attitude and practices of diabetic patients in the United Arab Emirates. PLoS One. 2013;8:e52857.

17. Göke R, Bader G, Dworak M. Real-life effectiveness and tolerability of vildagliptin and other oral glucose-lowering therapies in patients with type 2 diabetes in Germany. Diabetes Ther. 2014;5:183-191.

18. Nilsson PM, Brath H, Penfornis A, et al. Real life effectiveness and safety of vildagliptin compared with other OADs in European type 2 diabetes mellitus patients: results from the EDGE study. Diabetologia. 2014;57 Suppl 1:S1-S366.

19. Ahrén B, Mathieu C, Bader G, Schweizer A, Foley JE. Efficacy of vildagliptin versus sulfonylureas as add-on therapy to metformin: comparison of results from randomised controlled and observational studies. Diabetologia. 2014;57:1304-1307.

20. Mirzazadeh A, Baradaran HR, Haghdoost AA, Salari P. Related factors to disparity of diabetes care in Iran. Med Sci Monit. 2009;15: PH32-PH36.

21. Mahfouz EM, Awadalla HI. Compliance to diabetes self-management in rural El-Mina, Egypt. Cent Eur J Public Health. 2011;19: $35-41$.
22. Ligueros-Saylan M, Foley JE, Schweizer A, Couturier A, Kothny W. An assessment of adverse effects of vildagliptin versus comparators on the liver, the pancreas, the immune system, the skin and in patients with impaired renal function from a large pooled database of Phase II and III clinical trials. Diabetes Obes Metab. 2010;12:495-509.

23. Schweizer A, Dejager S, Foley JE, Couturier A, Ligueros-Saylan M, Kothny W. Assessing the cardio-cerebrovascular safety of vildagliptin: meta-analysis of adjudicated events from a large Phase III type 2 diabetes population. Diabetes Obes Metab. 2010;12:485-494.

24. Rathmann W, Kostev K, Gruenberger JB, Dworak M, Bader G, Giani G. Treatment persistence, hypoglycaemia and clinical outcomes in type 2 diabetes patients with dipeptidyl peptidase-4 inhibitors and sulphonylureas: a primary care database analysis. Diabetes Obes Metab. 2013;15:55-61.

25. Al-Arouj M, Hassoun AA, Medlej R, et al. The effect of vildagliptin relative to sulphonylureas in Muslim patients with type 2 diabetes fasting during Ramadan: the VIRTUE study. Int J Clin Pract. 2013;67: 957-963.

26. Hassoun A, Al-Arouj M, Ibrahim M. The effect of vildagliptin relative to sulphonylurea as dual therapy with metformin (or as monotherapy) in Muslim patients with type 2 diabetes fasting during Ramadan in the Middle East: the VIRTUE study. Available from: http://www researchgate.net/publication/259389442_The_First_Clinical_Congress_of_the_Gulf_Chapter_of_the_American_Association_of_Clinical_Endocrinologists_October_3_rd_-5_th_2013_St_Regis_Hotel_ Abu_Dhabi_United_Arab_Emirates. Accessed January 8, 2015.
Vascular Health and Risk Management

\section{Publish your work in this journal}

Vascular Health and Risk Management is an international, peerreviewed journal of therapeutics and risk management, focusing on concise rapid reporting of clinical studies on the processes involved in the maintenance of vascular health; the monitoring, prevention and treatment of vascular disease and its sequelae; and the involvement of

\section{Dovepress}

metabolic disorders, particularly diabetes. This journal is indexed on PubMed Central and MedLine. The manuscript management system is completely online and includes a very quick and fair peer-review system, which is all easy to use. Visit http://www.dovepress.com/ testimonials.php to read real quotes from published authors. 